\title{
Comparative Study of the Localisation of the Epidural Space Using Epidural Balloon Technique versus Saline Filled Syringe Technique - A Prospective, Randomised Study
}

\author{
Dr. Manali Nadkarni ${ }^{1}$, Dr. R. D. Patel ${ }^{2}$, Dr. Sushil Kumar Chouhan ${ }^{3}$, \\ Dr.Atul Walzade ${ }^{4}$ \\ ${ }^{\text {I}}$ (Additional Professor, Department Of Anaesthesiology, Seth G. S. Medical College \& K.E.M. Hospital, India) \\ ${ }_{2}^{2}$ (Professor, Department Of Anaesthesiology, Seth G. S. Medical College \& K.E.M. Hospital, India) \\ ${ }_{3}^{3}$ (Ex Postgraduate Student, Department Of Anaesthesiology, Seth G. S. Medical College \& K.E.M. Hospital, \\ India) \\ ${ }^{4}$ (Assistant Professor, Department Of Anaesthesiology, Seth G. S. Medical College \& K .E .M. Hospital, India)
}

\begin{abstract}
:
Background and aims:The success of epidural anaesthesia begins with proper identification of epidural space. There are different techniques used for identification of the epidural space. In this study we compare the localisation of epidural space using epidural balloon technique versus normal saline filled syringe technique .Method: 200 adult patents of ASA I \&II status posted for elective lower abdominal and lower limb surgery were randomly allocated in two groups of 100 each. After taking consent and IV access epidural anaesthesia was given to the patients. In group I epidural space was localised by epidural balloon technique while in group II epidural space was located by normal saline filled syringe technique using the loss of resistance method. The parameters studied were time taken for epidural space localisation, the number of attempts, the ease of passing the epidural catheter after localisation, success or failure rate of the localisation of the epidural space by resident anaesthesiologistsand the incidence of complications if any. Student unpaired ' $t$ ' test and chi square test were used for statistical analysis.

Results:90\% cases from group I(epidural balloon group) had localisation of epidural space in first attempt which was significantly more as compared to $78 \%$ in group II(saline filled syringe group). Mean duration of procedure in group I was 20.74 seconds which was significantly less as compared to 43.54 seconds in group II. $12 \%$ of cases from group II had complications during the procedure which was significantly more as compared to none from group $I$.

Conclusion: For epidural space localisation epidural balloon technique is better than saline filled syringe technique in terms of number of attempts, mean duration of procedure, incidence of complications and ease of technique.
\end{abstract}

Keywords: Epidural balloon, epidural anaesthesia, localisation of epidural space, loss of resistance technique.

\section{Introduction}

Epidural anaesthesia is a necessary skill for anaesthesiologists as it provides satisfactory operating conditions and also provides a very effective means of postoperative analgesia and has a low incidence of side effects.

Despite the manifest advantages of epidural block, many anaesthesiologists, especially those in peripheral centres do not employ the technique as they feel that the apparent difficulty in locating the epidural space as too great. Also they are afraid of inadvertent puncture of dura matter and unintentional injection of local anaesthetics into subarachnoid, subdural or intravascular spaces. This has resulted in an increasing apprehension over the use of this excellent technique of anaesthesia.

Identification of the precise moment when the needle is advanced into the epidural space decreases the likelihood that the needle will puncture the dura matter. Many studies have been conducted to determine the ways of and means to achieve better success rate by the use of various techniques for recognition of epidural space. Identification of epidural space should be simple, safe and incorporate easily available materials and monitoring devices.

A variety of techniques and modifications for detection of epidural space have been described including the hanging drop technique described by Gutierrez ${ }^{1}$, the running infusion drip described by Baraka ${ }^{2}$, the Oxford detector ${ }^{3}$, ultrasonic localisation, etc. ${ }^{4}$ Loss of resistance technique as described by Dogliotti ${ }^{5}$ is commonly used method for identification of epidural space. 
In this study we have attempted to evaluate the midline approach for the detection of epidural space by two different techniques. We have used epidural balloon(fig.1) technique and normal saline filled syringe technique to find out a simple and safe method for identification of epidural space.

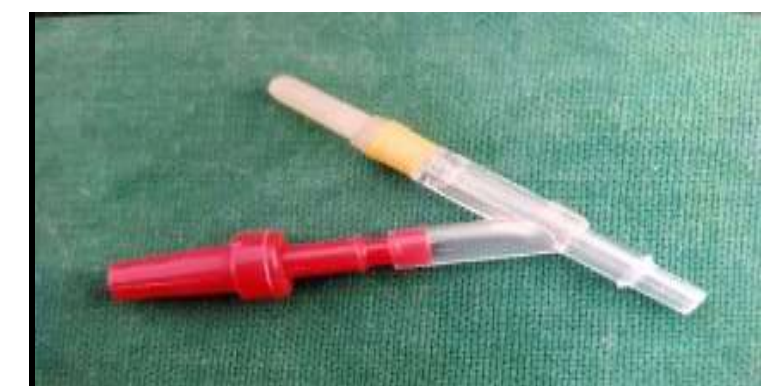

Figure 1. Epidural balloon(manufactured by VYGON)

\section{Aims and objectives}

The aim of the study was to compare the efficacy of epidural space localisation in $\mathrm{ASA}^{6}$ I \& IIpatients using epidural balloon technique versus saline filled syringe technique on the basis of the number of attempts and time taken for localisation of epidural space, the ease of passing the epidural catheter after localisation of the epidural space, the success and failure rate of localisation of epidural space and comparing any significant complications between the two approaches of epidural space localisation by resident anaesthesiologists.

\section{Materials and methods}

All consenting ASA status I \& II patients above 18 years of age posted for elective lower abdominal and lower limb surgeries in whom epidural catheter insertion was indicated were included in this study. Patients who were unwilling to participate or were having any neurological condition, spine deformities, local skin sepsis, hypersensitivity to local anaesthetics, bleeding or coagulation disorders and those on anticoagulant therapy and pregnant patients were excluded from the study.

\section{Conduct of the study}

After the institutional ethics committee approval this study was conducted in a total of 200 patients posted for elective lower abdominal and lower limb surgeries. They were randomly assigned in two groups of 100 each. In group I, epidural balloon technique was used to locate the epidural space. In group II, normal saline filled syringe technique was used to locate the epidural space. The procedure was done by trainee resident anaesthesiologists under supervision of senior anaesthesiologists.

After obtaining informed consent and premedication, intravenous access was taken and standard monitors were attached.

Under strict aseptic precautions with the patient in the sitting position, after local infiltration with $2 \mathrm{cc}$ of $2 \%$ inj. Lignocaine, a 16 G Touhy needle was introduced through the L2- L3 intervertebral space via midline approach. In group I after advancing the Touhy needle from the skin to supraspinous ligament, the epidural balloon was attached to the hub of the epidural needle (fig. 2) and the balloon was inflated with $2 \mathrm{ccs}$ of air (fig. 3). The epidural needle was advanced deeper (fig. 4) and as the needle passed through the ligamentum flavum and entered the epidural space the balloon got deflated (fig. 5). The time from the inflation of the balloon to the entering of the epidural space was noted.

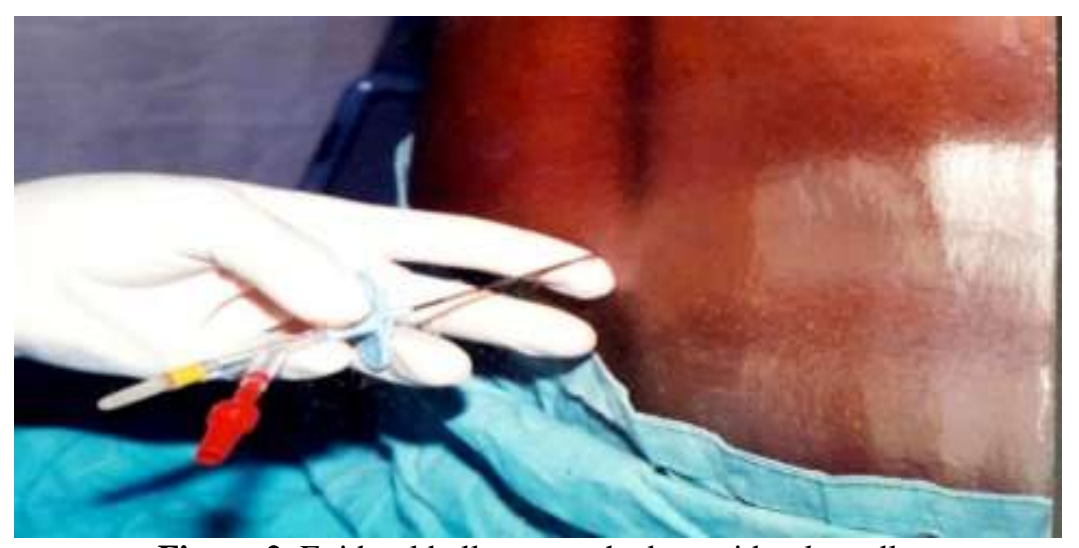

Figure 2. Epidural balloon attached to epidural needle 


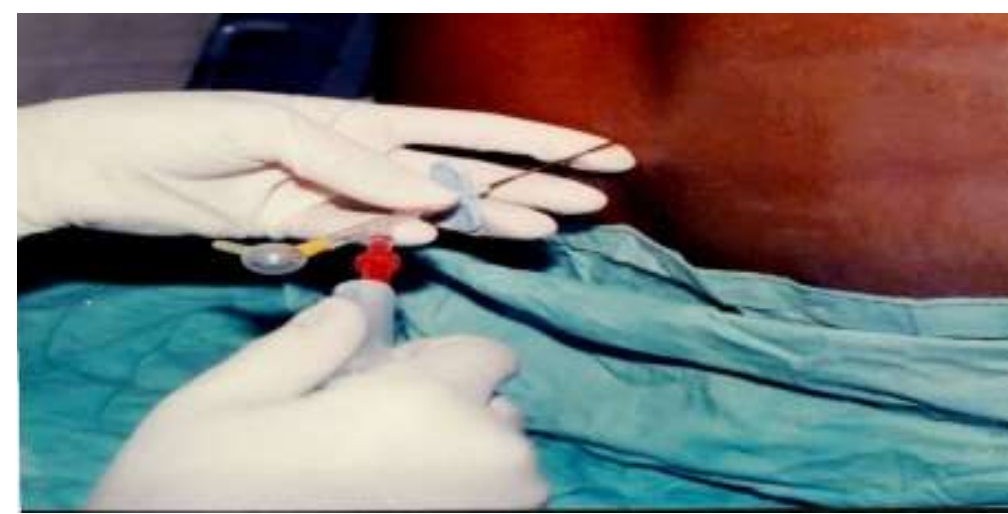

Figure 3. Epidural balloon inflated with air

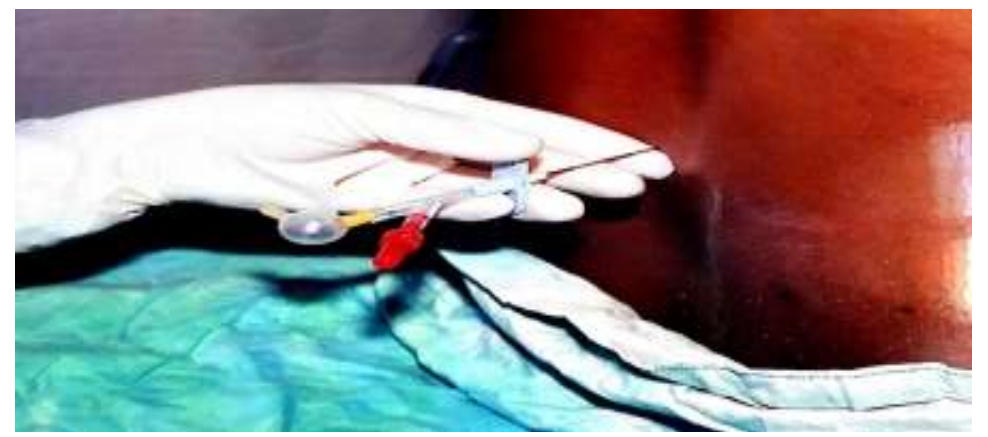

Figure 4. Epidural needle advanced slowly

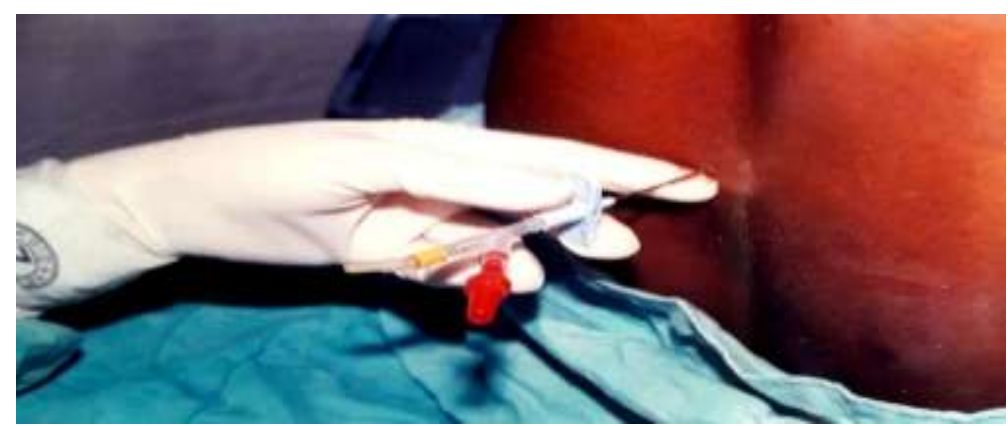

Figure 5. Epidural balloon deflates on entering epidural space

In group II after advancing the Touhy needle from the skin into supraspinous ligament, a 10 ccs syringe filled with 4 ccs of normal saline was attached to the hub of the needle and the epidural needle was advanced deeper slowly with continuous checking for bounce of the saline filled syringe. At the point where there was a loss of bounce, $2 \mathrm{cc}$ of saline was injected after negative aspiration and the epidural space was confirmed with the smoothness of the flow.

The time taken for localisation of the epidural space, the number of attempts taken to locate the space, the depth of the epidural space, the ease of passing the epidural catheter and any complications arising because of the procedure were noted.

\section{Observation and results}

In this study, for each parameters of both the groups, mean and standard deviation were calculated by using the computer software package 'Epistat'.

To find out the significant difference between the two groups, student unpaired ' $t$ ' test was used and chi square test for quantitative data. The level of significance were 0.05 . 
TABLE IDemographic Profile Of Patients

(a) By student ' $t$ ' test

\begin{tabular}{|l|l|l|}
\hline Parameters & Group I & Group II \\
\hline No. of patients & 100 & 100 \\
\hline Age(in years) ${ }^{(\mathbf{a})}$ & & \\
\hline Mean & 51.28 & 48.71 \\
\hline SD & 19.57 & 18.64 \\
\hline Range & $18-60$ yrs & $18-60$ yrs \\
\hline Weight(in kgs) ${ }^{(\mathbf{a})}$ & & \\
\hline Mean & 58.87 & 59.58 \\
\hline SD & 14.70 & 12.68 \\
\hline Range & $35-100$ & $35-95$ \\
\hline Sex(\%) & \\
\hline Male & & \\
\hline Female & 60 & 67 \\
\hline & 40 & 33 \\
\hline
\end{tabular}

(b) By chi square test

$\mathrm{p}>0.05$ not significant

This table reveals that mean age of the patients was 51.28 years in group I(epidural balloon) and 48.71 years in group II(saline filled syringe)which was the same and difference was not significant. The mean weight of the patients in both groups were comparable. $60-67 \%$ of total cases were males in both the groups.

TABLEIIComparison of number of attempts by resident anaesthesiologists

\begin{tabular}{|c|c|c|c|c|c|c|}
\hline \multirow[t]{3}{*}{ Post of anaesthesiologist } & \multicolumn{6}{|c|}{ Number of attempts } \\
\hline & \multicolumn{3}{|c|}{ Group I } & \multicolumn{3}{|c|}{ Group II } \\
\hline & 1 & 2 & 3 & 1 & 2 & 3 \\
\hline First year resident & $\begin{array}{l}50 \\
(92.6 \%) \\
\end{array}$ & $\begin{array}{l}4 \\
(7.4 \%)\end{array}$ & - & $\begin{array}{l}43 \\
(76.8 \%) \\
\end{array}$ & $\begin{array}{l}9 \\
(16.1 \%)\end{array}$ & $\begin{array}{l}4 \\
(7.1 \%) \\
\end{array}$ \\
\hline Second year resident & $\begin{array}{l}29 \\
(87.9 \%)\end{array}$ & $\begin{array}{l}4 \\
(12.1 \%)\end{array}$ & - & $\begin{array}{l}21 \\
(77.8 \%) \\
\end{array}$ & $\begin{array}{l}5 \\
(18.5 \%) \\
\end{array}$ & $\begin{array}{l}1 \\
(3.9 \%)\end{array}$ \\
\hline Third year resident & $\begin{array}{l}11 \\
(84.6 \%)\end{array}$ & $\begin{array}{l}1 \\
(7.7 \%)\end{array}$ & $\begin{array}{l}1 \\
(7.7 \%)\end{array}$ & $\begin{array}{l}14 \\
(82.3 \%)\end{array}$ & $\begin{array}{l}2 \\
(11.8 \%)\end{array}$ & $\begin{array}{l}1 \\
(5.9 \%)\end{array}$ \\
\hline Total & $\begin{array}{l}90 \\
(90 \%)\end{array}$ & $\begin{array}{l}9 \\
(9 \%)\end{array}$ & $\begin{array}{l}1 \\
(1 \%)\end{array}$ & $\begin{array}{l}* 78 \\
(78 \%)\end{array}$ & $\begin{array}{l}16 \\
(16 \%)\end{array}$ & $\begin{array}{l}6 \\
(6 \%)\end{array}$ \\
\hline
\end{tabular}

By chi square test $* \mathrm{p}<0.05$ significant

This table reveals that $90 \%$ of total cases from group I (epidural balloon) had localisation of epidural space in first attempt which was significantly more as compared to $78 \%$ in group II (saline filled syringe). $22 \%$ of total cases from group II required 2-3 or more attempts as only $10 \%$ in group I.

Table IIIComparison of duration of procedure between group I and group II

\begin{tabular}{|l|l|l|l|l|}
\hline Groups & \multicolumn{2}{|l}{$\begin{array}{l}\text { Mean duration of procedure according to post of anaesthesiologist(in } \\
\text { seconds) }\end{array}$} & \begin{tabular}{l} 
Mean $\begin{array}{l}\text { duration of } \\
\text { procedure } \\
\text { grou }\end{array}$ \\
\cline { 2 - 5 } \\
\cline { 2 - 5 } the
\end{tabular} \\
\hline I & First year resident & Second year resident & Third year resident & $20.74 \pm 18.55$ \\
\hline II & $21.05 \pm 9.63$ & $19.70 \pm 8.09$ & $18.0 \pm 8.01$ & $* 43.54 \pm 16.81$ \\
\hline
\end{tabular}

$* \mathrm{P}<0.05$ significant

Mean duration of procedure among group I was 20.74 seconds which was significantly less as compared to 43.54 seconds in group II.

Third year resident anaesthesiologist took significantly less time as compared to first year and second year resident anaesthesiologist in group II. In group I, third year resident anaesthesiologists took 18 seconds as compared to 21.05 seconds by first year resident anaesthesiologists which was same.

TABLEIV Profile of epidural catheter placement

\begin{tabular}{|l|l|l|l|l|}
\hline \multirow{2}{*}{ Procedure } & \multicolumn{2}{|c|}{ Group I } & \multicolumn{2}{c|}{ Group II } \\
\cline { 2 - 5 } & No. of patients & \% & No. of patients & \% \\
\hline Easy & 90 & 90 & 80 & 80 \\
\hline Difficult & 10 & 10 & 14 & 14 \\
\hline
\end{tabular}

By chi square test $\mathrm{p}>0.05$ not significant

Above data reveals that $14 \%$ of total cases from group II (saline filled syringe group) had difficulty during epidural catheter placement which was on higher side as compared to only $10 \%$ in group I (epidural balloon) but difference was not statistically significant. 
Comparative Study Of The Localisation Of The Epidural Space Using Epidural Balloon....

Table VComparison of complications during the procedure between group I and group II

\begin{tabular}{|l|l|l|l|l|}
\hline \multirow{2}{*}{ Complications } & \multicolumn{2}{|c|}{ Group I } & \multicolumn{2}{c|}{ Group II } \\
\cline { 2 - 5 } & Number & \% & Number & \% \\
\hline Blood on aspiration & Nil & - & 8 & 8 \\
\hline Dural puncture & Nil & - & 4 & 4 \\
\hline Total no. of patients & Nil & - & $* 12$ & 12 \\
\hline
\end{tabular}

By chi square test* $\mathrm{P}<0.05$ significant

This table shows $8 \%$ of patients in group II(saline filled syringe) had blood on aspiration and $4 \%$ had dural puncture as compared to not a single patient having complication in group I(epidural balloon). 12\% of patients from group II(saline filled syringe) had complications during procedure which was significantly more as compared to none from group I(epidural balloon).

\section{Discussion}

The epidural space is a region of loosely bound tissue, presenting little resistance to the propagation of hydrostatic pressure changes, enclosed in tissues that are highly resistant to pressure changes. Methods of identifying the epidural space depend in one way or another upon the recognition of the passage of the needle tip from the tissues of high resistance to those of low resistance.

The idea of passing a needle through 1.25 to 2.5 inches of tissues and then halting the point accurately in a narrow space a few millimetres wide seems daunting to the beginner, yet the procedure is much less difficult than would appear at first sight. Majority of failures are rooted in sloppy or inadequate technique.

Different types of devices for identifying the epidural space have been developed over the years. ${ }^{7}$ They were designed on the principle of demonstration of subatmospheric pressure in the epidural space or sudden loss of resistance ${ }^{7}$. Odom's indicator ${ }^{8}$, Macintosh's balloon ${ }^{9}$ and Brook's modification of the Odom's indicator ${ }^{10}$ are a $^{\text {and }}$ few of the devices working on the principle of subatmospheric epidural pressure. ${ }^{7}$

Massey Dawkins ${ }^{11}$ suggested three classes of epidural indicators - the tactile, the visual and the mechanical devices.

The tactile method where the thumb of the anaesthesiologist rests on the end of the piston of the syringe containing air or saline, depends for its success on the recognition of the sudden loss of resistance as the advancing needle point leaves the ligamentum flavum and travels freely in the epidural space.

The visual method depends for its success on the recognition of the fact that in $88.4 \%$ of cases a negative pressure exists in the epidural space ${ }^{12}$. The visual technique is both more accurate in regard to the avoidance of dural puncture and very much safer in the prevention of an accidental spinal e.g. hanging drop technique, Odom's indicator, Mustafa and Milliken's device.

In the mechanical device visual or tactile sense or both senses are replaced by mechanical force to reduce the failure rate. In this device the pressure of the anaesthesiologist's thumb on the piston can be replaced by a spring acting on the piston, a spring loaded trocar inside the needle or by a pressure balloon e.g. Zorraquin's bulb indicator, Macintosh spring loaded needle, Macintosh's extradural space balloon indicator, Ikle's syringe, Zelenka's balloon indicator, Brook's modification of Odom's indicator, Oxford epidural space detector, Ahmed El Mulla's device, Epidural balloon.

The epidural balloon (manufactured by VYGON) used in this study consists of - an inflatable balloon with a capacity of $5 \mathrm{ml}$ which is plugged into one end of the device, a one way valve through which the balloon is inflated and a free end of the device which attaches to the Touhy needle. This device is similar to Macintosh balloon, the only difference being that Macintosh balloon is inflated through its thick rubber neck, whereas one way valve is provided in this epidural balloon to inflate it.

Although the traditional loss of resistance technique to saline remains the choice of many experienced anaesthesiologists, one cause of failed epidural is false loss of resistance ${ }^{13}$. In some young adults the ligaments are quite soft and resistance to injection is not quite distinct. On the contrary in the elderly patients calcified ligaments may cause difficulty in locating the epidural space.

We studied a total number of two hundred patients with ASA physical status I \& II. All the patients were scheduled for elective lower abdominal or lower limb surgeries. (refer table I)

\section{- Number of attempts in localising epidural space (refer table II)}

Epidural puncture was accomplished at first attempt in ninety percent of patients in epidural balloon group which was significantly ( $\mathrm{p}<0.05$ significant) more as compared to seventy eight percent patients in saline filled syringe group. Twenty two percent patients in saline filled syringe group required two or more attempts as compared to ten percent patients in epidural balloon group. In study by Mustafa K. et $\mathrm{al}^{14}$ epidural puncture was accomplished at first attempt in $97.4 \%$ cases by using visual indicator. In $2.6 \%$ cases a second attempt was made. The possible explanation could be as follows. A firm braced grip on the needle and a slow controlled advance are among the first essential condition for the tactile technique. As saline in the syringe makes the device heavy, it is difficult for inexperienced anaesthesiologist to hold the needle in one hand only and apply 
pressure to the piston with the other hand. So the needle can deviate from the midline and enter the lumbar muscle even after locating the intervertebral space correctly. This causes false loss of resistance therefore more attempts needed to localise the epidural space. The epidural space balloon device being lighter than the saline filled syringe device allows the anaesthesiologist to use both the hands in guiding the needle.

\section{- Duration of procedure(refer table III)}

The mean duration of procedure (time taken from skin puncture with Touhy needle to localisation of epidural space) in epidural balloon group was $20.74 \pm 18.55$ seconds and in saline filled syringe group was $43.54 \pm 16.81$ seconds. Hence time taken to localise the epidural space using saline filled syringe technique was significantly more than the other group. Also the first and second year resident anaesthesiologists took longer time to localise the epidural space in saline filled syringe group as compared to epidural balloongroup. The mean duration of procedure by first year, second year and third year resident anaesthesiologists in epidural balloon group was $21.05 \pm 9.63 \mathrm{secs}, 19.7 \pm 8.09$ secs and $18 \pm 8.01$ secs respectively. Hence time taken to localise the epidural space by different level of resident anaesthesiologists was almost same in epidural balloon group. The reason is probably that the epidural space balloon device is much lighter than the saline filled syringe device and it enables the anaesthesiologist to use both the hands in guiding the needle instead of only one as with the tactile method. Therefore inexperienced anaesthesiologist also takes less time to localise the epidural space.

In saline filled syringe group time taken to localise the epidural space by first year and second year resident anaesthesiologists was $44.08 \pm 12.91$ secs and $48.18 \pm 22.82$ secs respectively which is significantly more than the time taken by third year resident anaesthesiologist which was $34.47 \pm 14.23$ secs. This may be because the saline filled syringe technique involves holding the needle in one hand only while the other hand applies pressure to the piston which the beginner may find difficult. Also the saline filled syringe is heavy to hold.

\section{- Epidural catheter placement (refer table IV)}

Epidural catheter placement was difficult in ten percent patients in epidural balloon group and fourteen percent patients in saline filled syringe group. This difference is statistically not significant. This could be attributed to difference in skill at different levels of resident anaesthesiologists during epidural catheter placement.

\section{- Complications during procedure (refer table V)}

$>$ Blood aspiration from epidural catheter

In epidural balloon group there was no case of blood on aspiration from epidural catheter as compared to eight patients in saline filled syringe group. Failure to judge exact point of entry into epidural space due tobulky device and inexperienced hand may be the cause of high incidence of venous puncture in saline filled syringe group. Whereas in epidural balloon group because of light weight of epidural balloon device the anaesthesiologist could use both the hands in guiding the needle into the epidural space and balloon got automatically deflated on entering the epidural space.

$>$ Dural puncture

Four percent of patients in group II (saline filled syringe) developed unintentional puncture of the dura while there was no incidence of dural puncture in group I( epidural balloon).

The problem with loss of resistance technique is that in elderly patients with partially ossified ligaments, the onward movement of the needle may not be checked in time. Consequently the incidence of dural puncture is double as compared to younger patients.

\section{- Success rate and failure rate}

Success rate was $100 \%$ in epidural balloon group as compared to $96 \%$ success rate in saline filled syringe group (tactile method). Ahmed El Mulla ${ }^{15}$ has reported $93.3 \%$ success rate with epidural balloon technique whereas success rate with loss of resistance technique was $66.67 \%$. He used Foley's catheter balloon to identify the epidural space. Mustafa K. et al ${ }^{14}$ has reported $100 \%$ success rate with his visual indicator. His visual indicator was simply a segment of the standard clear plastic intravenous extension tubing. Dawkins M. ${ }^{16}$ has reported $97 \%$ success rate with tactile method.

\section{From our study we conclude}

\section{Conclusion}

Time taken for localisation of the epidural space and number of attempts for localisation is significantly reduced when epidural balloon is used instead of saline filled syringe.

Success rate is higher with epidural balloon technique

The incidence of dural puncture and blood aspiration from epidural catheter is lower with epidural balloon technique. 
The success rate is high and the incidence of dural puncture is reduced when epidural balloon technique is used even by junior anaesthesiologist.

Thus from this study we can infer that the epidural space balloon technique is simple, safe and easy for correct location of the epidural space, especially for the inexperienced anaesthesiologists until they have the experience of sensing the puncture of ligamentum flavum.

\section{References}

[1]. J. A. Aldrete, O.A. Auad, V.P.Gutierrez, A.J.Wright, Alberto Gutierrez and the hanging drop, Regional anesthesia and pain medicine,30(4),2005,397-404.

[2]. Baraka A., Identification of the thoracic epidural space by the running infusion drip technique, Canadian journal of anaesthesia,48,2001,935-936.

[3]. Evans JM. The Oxford epidural space detector,Lancet 1982;ii:1433-1434

[4]. A. Wantman, N. Hancox and P.R.Howell, Techniques for identifying the epidural space: a survey of practice amongst anaesthetists in the UK, Anaesthesia, 61,2006,370-375.

[5]. A. Mario Dogliotti. Research and clinical observations on spinal anesthesia: with special reference to peridural technique.Anesthesia and Analgesia March April 1933,59-65.

[6]. ASA Physical status classification system- American Society of Anesthesiologists. www.asahq.org.

[7]. S. Jacob, E. Tierney, Anaesthesia, 52, 1997, 141-143.

[8]. Odoms C. B., Areview of Pages' epidural anaesthesia with a report of 100 cases, New Orleans Medical and Surgical Journal, 88, 1936, 618-627.

[9]. Macintosh R. R., Extradural space indicator, Anaesthesia, 5, 1950, 98-99.

[10]. Brooks W., An epidural indicator, Anaesthesia,12, 1957, 227-228.

[11]. Dawkins M. Identification of the epidural space,Anaesthesia,18, 1963,66-77.

[12]. Usubiaga J. E.,Wikinski J.A., Epidural pressure and its relation to the spread of anaesthetic solution in epidural space, Anesthesia and analgesia,40, 1967, 440.

[13]. Miller, Textbook of Anaesthesia, $8^{\text {th }}$ edition,1707-1709

[14]. Mustafa K. and Milliken R.A.; A simple device for the identification of the epidural space, Anesthesiology 57, 1982,330-332.

[15]. Ahmed F. El Mulla: Evaluation of a simple method for correct location of the epidural space for trainees, Alexandria journal of anaesthesia, 2,1999,19-27.

[16]. Dawkins C. J.M.; An analysis of complication of extradural and caudal block, Anaesthesia,24, 1969,554-563. 